\title{
Geleitwort des Dekans der Fakultät für Soziale und Politische Wissenschaften
}

\author{
Helmut Staubmann
}

Auch ohne einer Zahlenmagie zugetan zu sein, sind 40 Jahre Geschichte eines Universitätsinstitutes ein würdiger Anlass innezuhalten, um Bilanz zu ziehen und die großen Linien der Entwicklung, auch der zukünftigen, in den Blick zu nehmen. Aufgrund meines vorgerückten Alters qualifiziere ich mich quasi zum Zeitzeugen für die 40 Jahre Institut für Politikwissenschaft in Innsbruck, und so möchte ich aus meiner ganz persönlichen Erfahrung und mit Beobachtungen aus der Perspektive einer verwandten Disziplin einige Gedanken entwickeln.

Das Jubiläum führt uns zurück in das Jahr 1977. Ich war damals mitten im Studium der Soziologie an der Universität Wien. Zwei Jahre später kam ich als frisch gebackener Magister der Soziologie an das Institut für Höhere Studien (IHS) in Wien. Dort finden sich in meinen Erinnerungen die ersten Bezüge zur Innsbrucker Politikwissenschaft. Das IHS war von Paul Lazarsfeld und Oskar Morgenstern gegründet worden, um die in den USA erfolgten Entwicklungen moderner Sozialwissenschaften nach Österreich zu bringen. Mit dem IHS als außeruniversitärer Institution war die Erwartung verbunden, dass sich dieser neue Geist in der Folge an den Universitäten durchsetzen würde. Ich habe diese Umbruchs- und Aufbruchstimmung noch genau so erlebt. Und ein Name, der zu meiner Zeit am IHS noch präsent war, war der von Anton Pelinka. Anton Pelinka war bereits in der Frühzeit des IHS (1965/66) als Scholar für Politikwissenschaft am IHS und dann in den 70er Jahren auch als Assistent dort tätig. Paul Lazarsfeld — ich zitiere jetzt Anton Pelinka — war „einer der einflussreichsten Sozialwissenschafter des 20. Jahrhunderts". Das Zitat stammt aus seinem Beitrag in dem von mir mitherausgegebenen Band Kritische Theorie und Gesellschaftsanalyse, der Festschrift für Kollegen Max Preglau aus dem klar hervorgeht, dass Paul Lazarsfeld auch für ihn ein Vorbild und Wegbereiter für ein zeitgemäßes Wissenschaftsverständnis war.

Ich erinnere mich dann noch an die Reise zu meinen Dienstantritt, das war Herbst 1981, von Wien nach Innsbruck. Ein Absolvent der Universität 
Innsbruck und danach Politikwissenschaft-Scholar am IHS hat mich in seinem Auto mitgenommen und mich mit seinen Schilderungen quasi auf die Situation an der Universität Innsbruck eingestimmt. Er kam immer wieder auf „Anton“ zu sprechen, für mich damals eine etwas ungewöhnliche Benennung für den Vorstand eines Universitätsinstitutes, die aber ganz den Gepflogenheiten des Umgangs zwischen Lehrenden und Scholaren am IHS entsprach.

Für mich haben sich aus dieser Vorgeschichte zwei Linien verwoben, die mein Bild des Institutes für Politikwissenschaft prägten: Zum einen die wissenschaftliche Aufbruchsstimmung eines neuen und modernen Selbstverständnisses der Sozialwissenschaften und außerdem auch eine Veränderung der universitären Kultur. Die Politikwissenschaft, wie wir etwas verkürzt sagten, stand für eine Kultur kollegial-ebenbürtiger Beziehungen zu Studierenden und zu MitarbeiterInnen, einer Demokratisierung der universitären Organisation - alles Veränderungen, die uns in der Summe dem Ideal einer universitas im originären Wortsinne näher brachten.

Das universitäre Leben war in den letzten 40 Jahren dramatischen Veränderungen unterworfen. Die wichtigsten Veränderungen waren wohl die digitale Revolution und, damit verbunden, die Globalisierung. Wenn ich die Veränderungen etwas überzeichnen darf — in der Soziologie sagen wir dazu Bildung von Idealtypen, was entschieden besser klingt - , dann würde ich sagen: Es war eine Entwicklung von einer regionalen Schreibmaschinenwissenschaft hin zu einer global-kompetitiven Computer- und Internetwissenschaft. Veränderungen in der Umwelt der Universität führen aber keineswegs automatisch zu Veränderungen in der Organisation und im Leben der Universität, es bedarf dazu einer Kreativität und bewussten Anstrengung und hier, denke ich, hat das Institut für Politikwissenschaft Vorbildliches geleistet. Die Leistungen umfassend darzustellen ist hier nicht der Platz, aber ein Beispiel, das ich jetzt insbesondere aus der Perspektive unserer fakultären Bemühungen für eine neue Publikationskultur eindrucksvoll empfunden habe, ist der Transfer der SSCI gelisteten Österreichischen Zeitschrift für Politikwissenschaft als open-access Journal an die Universität Innsbruck. Wir hoffen, dass es der Österreichischen Zeitschrift für Soziologie gelingen wird, hier nachzuziehen.

Der Aufbau der Innsbrucker Politikwissenschaft als Fach, des Institutes und danach der Fakultät für Politikwissenschaft und Soziologie ist unbestritten das große Verdienst von Anton Pelinka. Ich erinnere mich an viele Details von Begegnungen, die freundschaftlich aufgeschlossen mir persönlich und meinem Fach gegenüber waren, auch den verantwortungsvollen Umgang und die Förderung junger KollegInnen für ihre wissenschaftliche Entwicklung und ihre Karriere habe ich in guter Erinnerung. Nun empfinde ich es als besonders ehrenvoll, dass ich als Dekan seine Nachfolge antreten durfte. 
Ein besonderes Anliegen ist es mir auch, die Leistungen der Nachfolger von Anton Pelinka als Institutsleiter zu würdigen. Nachdem ich selbst mehrere Jahre die Leitung des Soziologie-Institutes innehatte, weiß ich, dass die Institutsleitung eine aufopfernde Leistung für das Fach und unsere Universität darstellt. Max Weber hat in seiner berühmten Rede „Wissenschaft als Beruf“ resümierend gemeint: „Das akademische Leben ist also ein wilder Hazard". Was diese Worte wirklich bedeuten, weiß man erst, wenn man Institutsleiter war. Ferdinand Karlhofer hat 13 Jahre das Institut geleitet. Über diese, vornehm ausgedrückt, laboröse Aufgabe hinaus war er eine Säule in der wissenschaftlichen Produktivität des Fachbereiches und hat das Fach und damit unsere Fakultät ganz in der Tradition von Anton Pelinka auch in der medialen Öffentlichkeit vertreten.

Mit Martin Senn hat sich das Institut für Politikwissenschaft einen Institutsleiter gewählt, der, so die bisherige Erfahrung, die Tradition fortsetzt. Unter seiner Leitung muss das Institut für Politikwissenschaft sich der nunmehr beginnenden Zukunft der nächsten 40 Jahre stellen, eine $\mathrm{Zu}$ kunft, die eine mindestens ebenso radikale Veränderung mit sich bringen wird wie die vergangenen 40 Jahre. Wie die Zukunft aussehen wird, wissen wir nicht, wir sind ja Wissenschaftler und keine Propheten, aber sie wird gewiss jede Menge an Herausforderungen mit sich bringen.

Nun drängt es mich zum Abschluss, noch etwas Abstraktes anzumerken, sozusagen aus meiner Profession als soziologischer Theoretiker. Es liegt nahe bei einer Betrachtung der letzten 40 Jahre, die fundamentalen Veränderungen innerhalb und außerhalb des Wissenschaftsbetriebes in den Fokus zu nehmen, so wie man bei jedem Geburtstag eines Kindes staunt, um wie viele Zentimeter es wieder gewachsen ist. Wenn man beim Fluss aber nur das Fließen sieht, landet man in einer Einseitigkeit, der bereits der alte griechische Philosoph Heraklit zum Opfer gefallen ist. So wie ein Fluss, so baut auch unser universitäres Leben nämlich ganz entschieden auf Konstanten auf.

Die großen Veränderungen betrafen die Mittel unserer Arbeit und sie haben uns viele Vorteile gebracht. Über die Mittel, so wichtig sie sind, sollte man aber nicht auf die Zwecke vergessen und die haben sich in der Wissenschaft in der Zeit seit Platon oder Aristoteles nicht verändert. Hier sehe ich zwei Konstanten. Die eine ist die Verantwortung für die Studierenden, die sich uns anvertrauen. Die Quantität der Verantwortung lässt sich ermessen an der Zahl derer, die ein Studium für Politikwissenschaft absolviert haben, das sind mittlerweile mehr als 2.500 Absolventinnen und Absolventen. Eine weitere Konstante liegt im Prinzip der Bindung an wissenschaftliche Rationalität und damit an den Zentralbegriff jeder Wissenschaft, nämlich den der Wahrheit. Jacques Derrida hat in einem Vortrag an der Standford University einmal gemeint: „Die Universität macht die Wahrheit zum Beruf - und sie bekennt sich zur Wahrheit, sie legt ein Wahrheitsgelübde ab. Sie erklärt und gelobt öffentlich, ihrer uneingeschränk- 
ten Verpflichtung gegenüber der Wahrheit nachzukommen." Das sehe ich auch so.

Die große Frage aber, die wir damit zu lösen haben, lautet: Wie finden wir die Wahrheit? Die Antwort, die sich darauf in praktisch allen wissenschaftstheoretischen Positionen findet, lautet: Man muss die Wahrheit dort suchen, wo man sie am ehesten findet - ich erkläre das meinen Studierenden üblicherweise anhand des Schwammerl-Suchens - präziser müsste man aber sagen, man muss die Wahrheit dort suchen, wo man sie vermutet. Man nennt das dann in der Fachsprache Hypothesenbildung. Wer mich kennt, weiß, dass ich gewiss keinem postmodernen Relativismus das Wort rede, ich bin jedoch aufgrund langjähriger Erfahrung zur Auffassung gekommen, dass sich die Vermutung über den Verbleib der Wahrheit je nach Tageszeit unterschiedlich darstellt. Und am Abend pflege ich mich der Meinung der alten Römer anzuschließen, die ihre Vermutung mit der berühmten These zum Ausdruck brachten: veritas est in vino, besser bekannt in der korrekteren lateinischen Formulierung als: in vino veritas. In diesem Sinne: Ein Prosit auf unser Institut für Politikwissenschaft, seine Errungenschaften der vergangenen 40 Jahre und eine gewiss ebenso erfolgreichen Zukunft!

Univ.-Prof. Dr. Helmut Staubmann Dekan der Fakultät für Soziale und Politische Wissenschaften 